\title{
Volume plaquetário médio e doença cardiovascular
}

\author{
Mean platelet volume and cardiovascular disease
}

Andréa Elisabet Wendland1, ; Mariela Granero Farias²; Waldomiro Carlos Manfroi ${ }^{3,4}$

Unitermos
Plaquetas
Volume plaquetário médio
Doença cardiovascular
Infarto

\section{resumo}

As plaquetas têm importante papel no desenvolvimento do trombo intravascular, a maior causa de síndrome coronariana aguda (SCA). Após a erosão ou ruptura da placa aterosclerótica, a ativação das plaquetas é crucial nos eventos pró-trombóticos que levam ao infarto do miocárdio (IM). O aumento da reatividade plaquetária está associado à evolução do volume plaquetário. Plaquetas grandes são enzimática e metabolicamente mais ativas e apresentam alto potencial trombótico. O volume plaquetário médio (VPM) é um marcador da função plaquetária. Níveis elevados de VPM têm sido identificados como fatores de risco independentes para o IM em pacientes com doença cardíaca coronariana. No entanto, os valores biológico e prognóstico de níveis elevados de VPM ainda são controversos. Os novos analisadores hematológicos fornecem esse índice plaquetário como parte integrante do hemograma, não havendo custos adicionais para o laboratório, podendo ser utilizado como marcador precoce de risco de eventos cardiovasculares, associado a marcadores tradicionais. Neste estudo descrevemos o valor clínico do VPM na aterosclerose coronariana e seu papel como fator de risco para SCA.

\section{abstract}

Platelets play an important role in intravascular thrombus development, a major cause of acute coronary syndrome (ACS). After erosion or rupture of atherosclerotic plaque, platelets activation plays a crucial role in prothrombotic events leading to myocardial infarction. Increased platelets reactivity is associated with increased platelet volume. Large platelets are enzymatically and metabolically more active and have high thrombotic potential. Mean platelet volume (MPV) is a marker of platelet function. Elevated levels of MPV have been identified as an independent risk factor for myocardial infarction in patients with coronary cardiac disease. However, the biological and prognostic value of increased levels of MPV is still controversial. The new hematological analyzers provide the platelet index as part of the hemogram, without additional costs to the laboratory, and it may be used as an early risk marker of cardiovascular events in association with traditional markers. In this study we describe the clinical value of MPV in coronary atherosclerosis and its role as a risk factor for ACS.

\section{key words}

Platelets

Mean platelet volume

Cardiovascular disease

Infarction

1. Farmacêutica bioquímica da Unidade de Bioquímica e Imunoensaios do Serviço de Patologia Clínica do Hospital de Clínicas de Porto Alegre (HCPA); especialista em análises clínicas pela Pontifícia Universidade Católica do Rio Grande do Sul (PUCRS).

2. Farmacêutica bioquímica da Unidade de Hematologia do Serviço de Patologia Clínica do HCPA; especialista em Hematologia pela Universidade Federal do Rio de Janeiro (UFRI).

3. Professor titular da Faculdade de Medicina da Universidade Federal do Rio Grande do Sul (FM/UFRCS); professor do Programa de Pós-Graduação em Cardiologia da UFRCS.

4. Membro do Programa de Pós-Graduação em Cardiologia da UFRCS. 


\section{Introdução}

As plaquetas têm papel predominante na patogenia das síndromes coronarianas agudas (SCAs): angina instável, infarto do miocárdio (IM) e morte súbita ${ }^{(24,31)}$. Tem sido sugerido que a hiperreatividade e a ativação plaquetária local desempenham função causal nos eventos coronarianos agudos.

$\mathrm{Na}$ atualidade admite-se que o tamanho das plaquetas seja um indicador sensível de sua reatividade e que sua magnitude é determinante na formação do trombo intracoronariano em presença de ruptura da placa aterosclerótica ${ }^{(31)}$.

Plaquetas grandes são metabólica e enzimaticamente mais ativas que as pequenas ${ }^{(24)}$. Plaquetas grandes na circulação, refletidas pela elevação do volume plaquetário médio (VPM), levam a maior agregação e facilitam a formação do trombo $(14,40,41,43)$, mostrando ser um fator de risco na angina instável ${ }^{(14,20,23,39)}$. Além disso, esse índice está aumentado em pacientes com IM, acidente vascular cerebral (AVC) e diabetes mellitus (DM), e tem sido demonstrado ser um marcador preditivo de futuros eventos coronarianos adversos após episódio de infarto ${ }^{(39)}$. O VPM também está associado a aumentado risco de reestenose após angioplastia coronariana ${ }^{(2)}$.

O VPM é rotineiramente disponível na maioria dos laboratórios modernos e pode ser medido rapidamente a baixos custos $^{(39)}$, com algumas limitações relacionadas com a dificuldade de padronização e interferência de variáveis pré-analíticas ${ }^{(15,38)}$.

\section{Plaquetas}

Plaquetas são pequenos fragmentos subcelulares formados a partir do citoplasma dos megacariócitos da medula óssea(4, 18, 34). Circulam em uma concentração de $150.000-450.000 / \mathrm{mm}^{3(34)}$, com característica forma discoide $^{(18,34)}$. Da quantidade total de plaquetas no corpo, $70 \%$ estão presentes na circulação e $30 \%$, no baço ${ }^{(4,34)}$. Permanecem na circulação por uma média de $10 \operatorname{dias}^{(4,29,34)}$ e são removidas por células reticuloendoteliais no baço e no fígado ${ }^{(34)}$. São elementos celulares que aderem a uma área de injúria, sendo o principal componente do tampão hemostático inicial. Como são elementos críticos na manutenção da homeostasia normal, anormalidades no número ou função das plaquetas podem levar a ruptura nesses passos iniciais, resultando em tendência a sangramento ${ }^{(34)}$.

A contribuição das plaquetas para a homeostase está na formação do tampão hemostático primário, na secreção de importantes componentes para posterior recrutamento de plaquetas, na provisão de uma superfície adequada para que o processo de coagulação possa ocorrer, na liberação de promotores de reparação do endotélio e na restauração da arquitetura normal do vaso ${ }^{(34)}$.

As plaquetas normalmente circulam sem se aderir ao endotélio vascular normal. Quando ocorre dano à parede do vaso, produtos subendoteliais, como colágeno, são expostos, e as plaquetas, então, aderem-se a essas substâncias e formam um tampão hemostático efetivo ${ }^{(34)}$.

Como as plaquetas não apresentam núcleo ${ }^{(4,34)}$, muitas das suas características morfológicas e biológicas são determinadas pela mensagem genética de sua célula precursora, o megacariócito da medula óssea. Cada megacariócito produz entre mil e 2 mil plaquetas ${ }^{(18,35)}$, e as diferenças na morfologia e fisiologia são determinadas durante ou antes do processo de sua fragmentação $0^{(5)}$. Esse processo de diferenciação do megacariócito e produção plaquetária é controlado por fatores humorais produzidos em resposta ao consumo ou destruição de plaquetas. Um distinto e único aspecto do desenvolvimento do megacariócito é o processo de poliploidização nuclear. Durante diferenciação terminal, antes de acontecer fragmentação citoplasmática, os megacariócitos sofrem variado número de ciclos de endomitoses e ficam poliplóides ${ }^{(5)}$.

A trombopoietina (TPO) é o maior regulador do processo de desenvolvimento do megacariócito e de produção plaquetária ${ }^{(10,17,23,43)}$. Essa ação é provavelmente modulada por uma série de citocinas e fatores humorais estimuladores, como as interleucinas 3, 6 e11 (IL-3, IL-6, IL-11), o fator estimulador de colônia de granulócitos e macrófagos (GM-CSF) e a eritropoietina (EPO). Em situações que aceleram a hematopoiese, a maturação citoplasmática megacariocítica é mais rápida que a nuclear, e as plaquetas formadas têm volume maior e são metabolicamente mais ativas ${ }^{(42)}$.

As plaquetas são heterogêneas quanto a tamanho, densidade e reatividade ${ }^{(36)}$. $\mathrm{O}$ tamanho plaquetário não muda durante sua vida na circulação, e isso demonstra que a heterogeneidade no tamanho não é consequência da idade das plaquetas, mas é determinada durante a megacariocitopoiese e a trombopoiese ${ }^{(35,36)}$. Tem sido sugerido que um aumento no número de cromossomos no núcleo do megacariócito (ploidia) pode estar associado à produção de plaquetas grandes e hiperreativas ${ }^{(36)}$. 
O tamanho plaquetário é um marcador (e possivelmente determinante) da função plaquetária. Plaquetas grandes são potencialmente mais reativas e agregáveis, contêm mais grânulos densos, são metabólica e enzimaticamente mais ativas que as plaquetas pequenas e têm maior potencial trombótico ${ }^{(11,25)}$. Expressam maiores níveis de substâncias pró-trombóticas, tromboxane (TXA2), serotonina, betatromboglobulina ( $\beta$-TG) e proteínas de superfície prócoagulatórias como P-selectina e glicoproteína IIla ${ }^{(11,25,31)}$. Além disso, são associadas a diminuído tempo de sangramento, uma medida de função hemostática in vivo ${ }^{(25,27,35)}$.

\section{Volume plaquetário médio}

Os estudos sobre plaquetas limitavam-se, há algum tempo, à contagem de plaquetas $/ \mathrm{mm}^{3} \mathrm{e}$ às referências morfológicas sobre macroplaquetas, satelitismo e agregação plaquetária. Com o surgimento dos analisadores hematológicos na década de 1980, tornou-se possível a mensuração automática de vários parâmetros plaquetários ${ }^{(22)}$. Entre os novos índices vem merecendo destaque o VPM, por se tratar de uma variável biológica que determina função e atividade plaquetárias ${ }^{(2,19,33)}$.

Embora a avaliação cuidadosa da morfologia plaquetária em uma distensão sanguínea seja rápida e acurada, ela é enganosa para a estimativa do tamanho da plaqueta $^{(3,8)}$ (Figura), por isso a utilização do VPM determinado pelos analisadores hematológicos pode melhorar a descrição de várias desordens plaquetárias ${ }^{(3)}$.

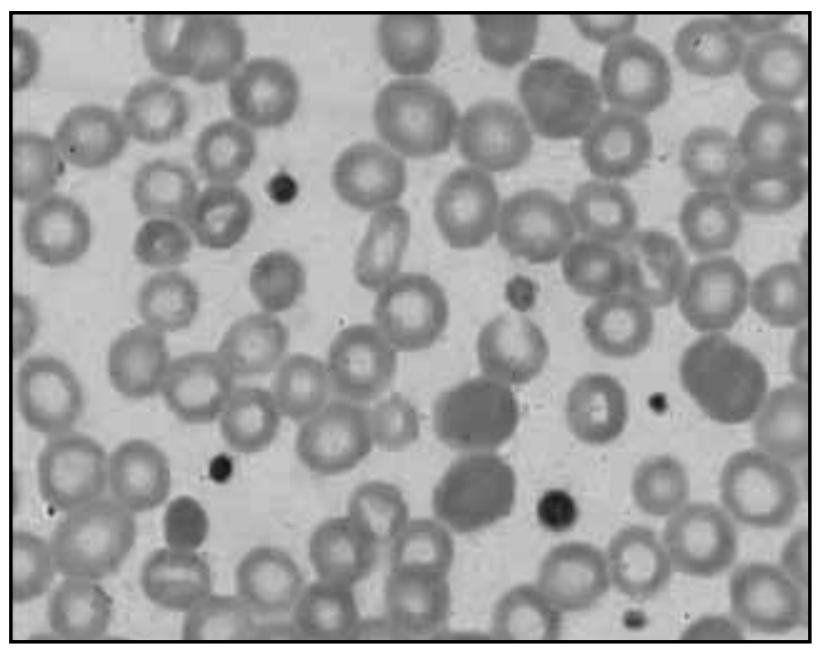

Figura - Distensão sanguínea corada com May-Crünwald-Giemsa (1.000x) mostrando macroplaquetas em um paciente com VPM de $12 \mathrm{fL}$ VPM: volume plaquetário médio.
Uma analogia inversa entre o VPM e a contagem de plaquetas pode ser encontrada, pois ocorre uma relação inversamente proporcional à ploidia do megacariócito, e o volume plaquetário não pode ser avaliado separadamente da contagem de plaquetas ${ }^{(6,43)}$. O volume plaquetário é relacionado com a função plaquetária e pode ser um índice mais sensível que o número de plaquetas como marcador de interesse clínico.

Muitos laboratórios ignoram a informação do VPM, gerada em analisadores hematológicos, pela dificuldade de padronização. Uma série de fatores, como o tipo de tecnologia utilizada, populações de pacientes e variáveis pré-analíticas como efeito dos anticoagulantes, temperatura e tempo de estocagem do material, causam interferência na sua determinação(15, 38). Em virtude desses problemas, é aconselhável que cada laboratório estabeleça seu próprio valor de referência ${ }^{(9,24)}$.

Estudos prévios relataram que os valores de VPM aumentam em um padrão tempo-dependente, devido ao inchaço plaquetário, quando o ácido etilenodiaminotetracético (EDTA) é usado como anticoagulante, entretanto um estudo recente demonstrou que esse aumento do tamanho das plaquetas é aproximadamente inferior a $0,5 \mathrm{fL}$, quando a análise é realizada dentro de 2 horas após a venipunctura ${ }^{(14,20)}$.

Há dados limitados sobre o efeito de terapia farmacológica na contagem e no tamanho das plaquetas. Pouco se sabe sobre os efeitos da aspirina e de outros inibidores de agregação plaquetária no VPM. Em um pequeno estudo com 30 pacientes, não foi encontrado efeito nos valores de VPM. Entretanto, ainda precisa ser avaliado se intervenções com inibidores de agregação plaquetária ou outras drogas poderiam ser benéficas para pacientes com elevado VPM(11).

Atualmente está claro que as plaquetas têm importante papel não só na trombose e na hemostasia, mas também em desordens caracterizadas por doenças vasculares, incluindo doença arterial coronariana (DAC) ${ }^{(14)}$.

Plaquetas grandes são mais ativas hemostaticamente e o volume plaquetário pode estar elevado tanto em pacientes com angina instável quanto naqueles com IM. Além disso, o VPM é um preditor de evento isquêmico posterior e de morte quando medido após $\mathrm{IM}^{(36)}$.

\section{VPM e ativação plaquetária na doença cardíaca}

As plaquetas desempenham importante função no desenvolvimento de trombo intravascular, a maior causa de $S C A^{(25,32)}$. Após erosão ou ruptura de uma placa ateros- 
clerótica numa artéria coronariana, a ativação plaquetária é crucial nos eventos pró-trombóticos, levando ao $\mathrm{IM}^{(11,25,37)}$.

Nos locais de turbulência (bifurcações, estenose ou lesão endotelial), a hemólise das hemácias libera quantidade suficiente de difosfato de adenosina (ADP) para mobilizar a saída de íons cálcio microtubulares e ativar as plaquetas. A agregação primária é reversível e a desativação das plaquetas advém do retorno dos íons cálcio citoplasmáticos para os microtúbulos plaquetários, mecanismo dependente da prostaciclina produzida pelo endotélio íntegro e normofuncionante ${ }^{(1)}$. A ruptura de placas vulneráveis modifica sua geometria e propicia a trombose mural[1, 13). O substrato trombogênico placa-dependente, a reologia vascular e a atividade sistêmica pró-coagulante influenciam a magnitude, a estabilidade do trombo e a gravidade da $\mathrm{DAC}^{(1)}$.

A injúria no vaso causada pela ruptura da placa expõe colágeno e fator de von Willebrand às plaquetas, as quais, então, aderem-se e sua ativação local, com subsequente agregação e recrutamento de mais plaquetas, estimula a formação do trombo ${ }^{(12)}$. Entretanto, por mais que mudanças na placa sejam pró-trombóticas, a presença de plaquetas grandes e mais reativas também contribui para a trombose ${ }^{(36)}$.

Plaquetas grandes na circulação, refletidas pela elevação do VPM, levam a maior agregação e facilitam a formação do trombo ${ }^{(14,40,41,43)}$, mostrando ser um fator de risco na angina instável ${ }^{(14,20,23,39)}$. Além disso, esse índice está aumentado em pacientes com IM, AVC e DM, além de ser um marcador preditivo de futuros eventos coronarianos adversos após episódio de infarto( ${ }^{(39)}$. O VPM também está associado a aumentado risco de reestenose após angioplastia coronariana ${ }^{(2)}$.

\section{VPM e infarto}

Vários estudos encontraram associação entre VPM e DAC ou ocorrência de um infarto agudo do miocárdio (IAM) ${ }^{(11,26)}$, mas Halbmayer et al.(16), avaliando pacientes com DAC aguardando por cirurgia cardíaca, não a encontraram. Uma provável explicação seria que somente a transição da forma estável para instável da DAC é acompanhada pela ativação da trombopoiese e produção de plaquetas grandes ${ }^{(36)}$. Um elevado VPM após IM também foi considerado fator de risco de IM recorrente ${ }^{(28)}$.

O VPM e a contagem de plaquetas foram medidos seriadamente em cem pacientes logo após IM e novamente sete semanas depois do evento. O VPM após IM foi significativamente maior que nos controles $(9,07$ vs.
$8,32 \mathrm{fL} ; p<0,001)$ e permaneceu elevado após seguimento clínico, apesar da significativa queda nos valores entre admissão e seguimento $(8,69 \mathrm{fL} ; p<0,001)^{(42)}$. A contagem plaquetária média na admissão foi significativamente menor que no grupo controle $(275.000$ vs. $\left.295.000 / \mathrm{mm}^{3} ; p<0,005\right)$. Não houve diferença significativa entre VPM e contagem de plaquetas entre pacientes que morreram durante o estudo e os que sobreviveram. Os achados defendem a ideia de que mudanças no tamanho plaquetário podem estar ligadas ao desenvolvimento de IM. Em parte, o VPM aumentado poucas horas após o IM deve ser derivado de plaquetas circulantes antes do surgimento dos sintomas ${ }^{(7)}$.

Osuna et al. ${ }^{(31)}$ investigaram a influência do VPM sobre risco de morte, isquemia recorrente e insuficiência cardíaca durante a fase hospitalar. Foram estudados 1.082 pacientes com IM classificados, de acordo com o VPM na admissão, em dois grupos: VPM $>9 \mathrm{fL}(n=443)$ e VPM $\leq 9 \mathrm{fL}$ $(n=639)$. Quando ajustado para todas as variáveis selecionadas ao ingresso, um VPM $>9 \mathrm{fL}$ se relacionou com aumento independente de risco combinado de morte, insuficiência cardíaca e episódios isquêmicos pós-infarto (razão de chance $[R C]=1,37$; intervalo de confiança [IC] $95 \%$ 1,04-1,8; $p=0,026$ ). Em valores absolutos, o tamanho plaquetário foi significativamente mais elevado nos pacientes que apresentaram evento final combinado do que naqueles que evoluíram sem complicações $(8,95 \pm 1,2 \mathrm{fL}$ vs. 8,78 $\pm 1,03 \mathrm{fL} ; p=0,009)$. O VPM $>9 \mathrm{fL}$ se associou a aumento significativo do risco de IC grave $(\mathrm{RC}=1,46$; IC 95\% 1,07-1,99; $p=0,01$ ), junto a uma tendência não significativa na frequência de angina pós-infarto ou reinfarto ( $\mathrm{RC}=1,35$, IC 95\% 0,98-1,87; $p=0,07$ ), não sendo observado efeito significativo sobre a mortalidade $(\mathrm{RC}=1,24 ; \mathrm{IC}$ $95 \% 0,87-1,75 ; p=0,22$ ).

Devido ao fato de a insuficiência cardíaca se relacionar diretamente com o tamanho do IM, é possível que a elevação do VPM condicione uma trombose coronariana mais oclusiva e persistente, a qual se traduziria em infartos de maior tamanho. Os resultados sugerem que um VPM elevado na fase aguda do IM é um preditor independente de risco de IC grave em curto prazo.

Outro estudo avaliou VPM e contagem de plaquetas em 981 pacientes com angina estável (AE), angina instável (Al) e dor torácica de origem não cardíaca. Pacientes com AE não tiveram diferença na contagem plaquetária em comparação com o grupo controle, mas tiveram um VPM significativamente aumentado $(8,7$ vs. 8,2 fL; $p<0,01)$. Em contraste, pacientes com Al apresentaram diminuída 
contagem de plaquetas $\left(245.000 / \mathrm{mm}^{3}\right)$ em comparação tanto com $\mathrm{AE}\left(262.000 / \mathrm{mm}^{3} ; p<0,05\right)$ quanto com grupo controle $\left(261.000 / \mathrm{mm}^{3} ; p<0,05\right)$. Além disso, o VPM foi significativamente maior que no grupo $A E(9,4 \pm 1,23 \mathrm{fL}$; $p<0,01)$. Pacientes com Al requerendo imediata angioplastia tiveram uma contagem ainda menor de plaquetas $\left(231.000 / \mathrm{mm}^{3}\right)$ e maior VPM $(10,4 \pm 1,03 \mathrm{fL} ; p<0,01) \mathrm{em}$ relação ao resto da população com $\mathrm{Al}$, com aumento, em média, de mais de $25 \%$ em comparação com a população controle. Isso indica que a Al pode estar associada ou ser precedida por uma elevação sistêmica na taxa de destruição plaquetária que não é completamente compensada por um aumento na taxa de produção plaquetária. Além disso, o consumo de plaquetas no sítio da lesão culpada pode contribuir para a formação do trombo arterial coronariano na $\mathrm{Al} \mathrm{e}$, dessa forma, para o aumento da taxa de destruição de plaquetas ${ }^{(32)}$.

A liberação de plaquetas grandes pela medula óssea pode ser interpretada como consequência do consumo de plaquetas no sítio da lesão coronariana. Desde que mudanças no tamanho plaquetário são determinadas na trombopoiese e as plaquetas circulam por 10 dias, é possível que plaquetas grandes estejam presentes na circulação ao se iniciarem os sintomas. Os dados sugerem que um VPM elevado contribui para o estado pró-trombótico nas SCAs ${ }^{(32)}$

Um estudo indiano encontrou valores de VPM significativamente mais elevados em pacientes com Al e IAM $(10,43 \mathrm{fL})$ em relação àqueles com DAC estável (VPM 9,37 fL) e ao grupo controle (VPM 9,2fL). Uma análise univariada dos quartis do VPM indicou um valor de mais de 9,6 fL como significativo fator de risco para desenvolvimento de $\mathrm{IM}^{(24)}$.

Estudo realizado na Turquia ${ }^{(25)}$ investigou o valor clínico do VPM na aterosclerose coronariana e a possibilidade de ser um fator de risco independente de IAM. Foram incluídos 200 pacientes, divididos em cinco grupos (AE, Al, IM com e sem elevação de ST e controles). O VPM estava significativamente aumentado em pacientes com IM $(11,75$ $\pm 1,07 \mathrm{fL})$ em comparação com controles $(10,89 \pm 0,69$ $\mathrm{fL} ; p<0,001), \mathrm{AE}(11,25 \pm 0,74 \mathrm{fL} ; p<0,05)$ e pacientes com dois $(11,65 \pm 0,99 \mathrm{fL} ; p<0,001)$ e três vasos doentes $(11,65 \pm 0,92 \mathrm{fL} ; p<0,001)$. Houve também elevação leve nos valores de VPM no grupo Al $(11,37 \pm 0,91 \mathrm{fL})$ e nos pacientes com um vaso doente $(11,26 \pm 0,99 \mathrm{fL})$, embora não significativo.

Um alto valor de VPM ( $\geq 12 \mathrm{fL}$ ) demonstrou a ocorrência de DAC com sensibilidade de $34 \%$ e especificidade de
$98 \%$, além de evidenciar risco de IM com sensibilidade de $46 \%$ e especificidade de $87 \%$, igual à demonstrada pela fração $M B$ da creatinofosfoquinase (CK-MB) e maior que a demonstrada pela troponina I (Tnl) (70\%).

Yilmaz et al. ${ }^{(40)}$ investigaram se a agregação plaquetária indicada pelo VPM elevado está aumentada em pacientes com trombo no ventrículo esquerdo após IM. Dos 124 pacientes diagnosticados com IAM em parede anterior e tratados com trombolíticos, aspirina e heparina, $34,5 \%$ foram diagnosticados como tendo trombo mural (grupo 1) e 65,5\% sem trombo detectável no ecocardiograma (grupo 2). Não houve diferença entre os grupos com respeito a idade, sexo, período de administração de heparina ou fatores de risco coronariano convencionais. O grupo 1 apresentou valores significativamente maiores de VPM em relação ao grupo $2(9,4 \pm 0,9 \mathrm{fL}$ vs. $8,7 \pm$ $0,5 \mathrm{fL} ; p<0,001)$.

O maior nível de VPM nos pacientes com trombo mural em relação àqueles sem pode refletir plaquetas mais reativas na circulação, levando a maior agregação, particularmente quando uma estase ocorreu, facilitando a progressão da formação do trombo mural.

\section{VPM e AVC}

Um estudo multicêntrico ${ }^{(14)}$ e transversal fez a primeira descrição da relação entre VPM e severidade clínica e eventos cerebrovasculares isquêmicos agudos. Analisou a relação entre o VPM e a gravidade do AVC após uma semana em 76 pacientes com AVC isquêmico agudo. Indivíduos no maior quintil do VPM apresentaram risco duas vezes maior de sofrer AVC grave quando em comparação com pacientes no menor quintil ( $\mathrm{RC}=2,6$; IC 95\% 1,6-4,1; $P<0,001)$. Essa associação permaneceu significativa após ajuste para possíveis confundidores ( $R C=2,2 ;$ IC 95\% 1,2-4; $p=0,013$ ). Os resultados indicam que elevado VPM está associado a pior desfecho para eventos cerebrovasculares isquêmicos agudos, independentemente de outros parâmetros clínicos. Foi observada pequena, mas significativa, correlação negativa entre VPM e contagem plaquetária média $(r=-0,262$; $p<0,001)$. A contagem plaquetária não foi significativamente associada à severidade do AVC.

Devido à vida média das plaquetas ser de aproximadamente 10 dias, é improvável que o seu tamanho no momento da dosagem tenha sido afetado pelo evento vascular agudo. Mais provavelmente os resultados sugerem que pacientes que sofreram um AVC grave já possuíam elevado VPM, refletindo alta reatividade plaquetária antes da ocorrência do evento ${ }^{(14)}$. 
Outro estudo investigou a associação entre VPM e risco de AVC entre uma coorte de 3.134 participantes do Perindopril Protection Against Recurrent Stroke Study (PROGRESS), que foram acompanhados por $\pm 3,9$ anos. O VPM foi positivamente associado a risco de AVC, com aumentado risco relativo de $11 \%$ (IC 95\% 3\%-19\%) de AVC por aumento de $\mathrm{fL}$ de VPM. Esse estudo é o primeiro a mostrar, em um delineamento prospectivo, uma associação positiva entre VPM e risco de AVC. A associação foi independente de outros determinantes estabelecidos ${ }^{(2)}$.

Há relatos de que pacientes que evoluem mal após AVC (morte ou dependência) possuem elevação significativa do VPM na fase aguda e tendem a ter menor contagem plaquetária em relação àqueles que evoluem melhor (independência) $)^{(35)}$. Também foi demonstrado que o VPM permaneceu elevado três meses após o AVC. O significado disso ainda precisa ser estabelecido, mas já que o VPM medido seis meses depois do IM prediz eventos coronarianos recorrentes e mortalidade por todas as causas, um persistentemente elevado VPM após AVC isquêmico pode estar associado a evento vascular recorrente e morte ${ }^{(35)}$.

\section{VPM como marcador de reestenose}

As plaquetas desempenham papel crucial no remodelamento vascular após angioplastia coronariana percutânea transluminal (PTCA) ${ }^{(39)}$.

À parte do desenvolvimento do trombo no sítio do trauma induzido por balão, as plaquetas também são fundamentais no processo de proliferação vascular neointimal. Estudos experimentais têm demonstrado que constituintes dos alfa-grânulos das plaquetas são localmente liberados nas paredes íntima e média. Pelo fato de as plaquetas maiores serem hemostaticamente mais reativas que as menores $\mathrm{e}$ conterem mais grânulos densos e alfa-grânulos, é provável que a associação entre tamanho plaquetário e reestenose depois da primeira PTCA bem-sucedida dê-se por meio da aumentada exposição a fatores de crescimento derivados de plaquetas (PDGF), na parede do vaso lesado, que estão relacionados a processo de proliferação da célula muscular lisa, desenvolvimento de hiperplasia intimal e posterior reestenose ${ }^{(39)}$.

Um estudo retrospectivo investigou a influência do VPM na reestenose após PTCA. Foram incluídos 174 pacientes submetidos a PTCA eletiva, seguidos por seis meses. De acordo com os angiogramas no seguimento, os pacientes foram divididos em dois grupos: reestenose $(n=74)$ e não reestenose $(n=100)$. Ambos os grupos foram comparados em função dos parâmetros hematológicos de rotina pré- procedimento, que incluíam, entre outros, VPM e contagem de plaquetas ${ }^{(39)}$.

O VPM estava significativamente aumentado no grupo com reestenose em relação ao sem ela $(8,75 \pm 0,99 \mathrm{fL} v$ s. $8,04 \pm 0,74 \mathrm{fL} ; p<0,001)$. A contagem plaquetária se correlacionou inversamente com o VPM $(r=-0,36 ; p<0,01)$ e foi significativamente menor no grupo com reestenose em relação ao sem. Essa relação inversa pode indicar que aumentada atividade plaquetária e consumo antes da PTCA são seguidos por aumento no tamanho, compensando uma diminuição da contagem de plaquetas.

Pacientes com valores de VPM pré-procedimento $>8,6 \mathrm{fL}$ exibiram um risco 3,1 vezes maior de reestenose após PTCA que aqueles com valores de $\mathrm{VPM}<8 \mathrm{fL}$. Pacientes que desenvolveram reestenose dentro dos três primeiros meses após PTCA $(n=32)$ apresentaram valores de VPM significativamente mais elevados que aqueles com evidência de reestenose após esse período $(n=42)(9,11 \pm 0,96 \mathrm{fL}$ vs. $8,48 \pm 0,93 \mathrm{fL} ; p<0,01)^{(39)}$.

$O$ VPM parece ser um marcador de reestenose coronariana em pacientes submetidos a PTCA. Pacientes com altos valores de VPM pré-procedimento poderiam ter benefício com uma terapia antiplaquetária intensiva após intervenções coronarianas. Em adição, uma significativa correlação entre VPM e incidência relacionada ao tempo de restenose angiográfica foi aparente, sugerindo que o VPM é particularmente um marcador de reestenose precoce ${ }^{(39)}$.

Outro estudo procurou determinar o valor prognóstico do VPM para reperfusão angiográfica e mortalidade em seis meses em pacientes com IM com elevação de segmento ST tratados com intervenção percutânea coronariana primária ( $\mathrm{PCl})$. O VPM foi medido antes da $\mathrm{PCl}$ em 398 pacientes consecutivos, seguidos por mais de seis meses. O fenômeno de no-reflow foi significativamente mais frequente em pacientes com alto VPM ( $\geq 10,3 \mathrm{fL}$ ), valor de corte determinado pela curva receive operator caracteristic curve (ROC), comparado com aqueles com baixo VPM $(<10,3 \mathrm{fL})$ $(21,2 \%$ vs. $5,5 \% ; p<0,0001)$. Análise de sobrevivência de Kaplan-Meier demonstrou taxa de mortalidade em seis meses de $12,1 \%$ em pacientes com alto VPM vs. 5,1\% no grupo com baixo VPM. Depois de ajuste para características basais, elevado VPM permaneceu como forte preditor independente de no-reflow ( $\mathrm{RC}=4,7$, IC 95\% 2,3-9,9; $p<0,0001$ ) e mortalidade ( $R C=3,2$, IC 95\% 1,1-9,3; $p=0,0084$ ). Houve correlação negativa fraca, mas significativa, entre VPM e contagem de plaquetas $(r=-0,211 ; p<0,0001)^{(20)}$.

O VPM manteve uma contribuição independente na avaliação de risco de no-reflow, apesar dos valores de Tnl. 
O VPM alto na admissão foi associado a significativo risco de desenvolver no-reflow, tanto em pacientes com Tnl positiva quanto negativa. Além disso, o VPM médio foi significativamente maior nos 29 pacientes que morreram $(10,4 \pm 0,85 \mathrm{fL})$, em comparação com os 359 sobreviventes $(9,96 \pm 0,9 \mathrm{fL} ; p=0,0134)$.

Os resultados do estudo defendem a ideia de que plaquetas desempenham importante papel na fisiopatologia de no-reflow e sugerem que o VPM pode ser considerado um útil e independente marcador hematológico, permitindo identificação precoce de pacientes que estão em maior risco de prejudicada reperfusão após $\mathrm{PCl}^{(20)}$.

\section{Conclusão}

As plaquetas desempenham importante função na patogênese das SCAs e, quanto maiores, mais ativas e agregáveis, levando à formação do trombo intravascular, que é a maior causa das SCAs.

Valores mais elevados de VPM caracterizam pacientes com IM e Al quando em comparação com aqueles com AE e dor torácica de origem não cardíaca. Além disso, elevado
VPM tem sido reconhecido como fator de risco independente para AVC.

A gênese das alterações na função plaquetária é desconhecida. Como o ciclo de vida das plaquetas é limitado em 10 dias, qualquer estímulo ou alteração metabólica responsável pela ativação das plaquetas poderia ser recente ou estar acontecendo.

O VPM é uma medida indireta da reatividade plaquetária, podendo refletir mudanças no nível de estimulação ou na taxa de produção plaquetária.

O fundamento lógico para identificação de pacientes de alto risco é reduzir o risco de eventos vasculares pela administração de tratamento antiplaquetário apropriado e efetivo. Pacientes com plaquetas maiores podem facilmente ser identificados durante análise hematológica de rotina e poderiam possivelmente se beneficiar de tratamento preventivo.

O VPM é gerado a partir de analisadores hematológicos como parte integrante do hemograma, por isso não implica custos adicionais para o laboratório. Por se tratar de um método simples e econômico, que indica ativação plaquetária, esse índice poderia ser utilizado como marcador precoce de risco de eventos cardiovasculares, associado a marcadores tradicionais, além de adicionar informação prognóstica útil para pacientes com história de DCV.

\section{Referências}

1. ARMAGANIJAN, D. Ativação plaquetária nas formas distintas da doença arterial coronariana. Arq Bras Cardiol, v. 87, p. 401-2, 2006.

2. BATH, P. et al. Association of mean platelet volume with risk of stroke among 3,134 individuals with history of cerebrovascular disease. Stroke, v. 35, p. 622-6, 2004.

3. BESSMAN, J. D. Automated blood counts and differentials: a pratical guide. Johns Hopkins University Press, 1986. p. $57-83$

4. BRIGGS, C.; HARRISON, P.; MACHIN, S. I. Continuing developments with the automated platelet count. Int Jnl Lab Hem, v. 29, p. 77-91, 2007.

5. BROWN, A. S. et al. Megakaryocyte ploidy and platelet changes in human diabetes and atherosclerosis. Arterioscler Thromb Vasc Biol, v. 17, p. 802-7, 1997.

6. BUTKIEWICZ, A. M. et al. Platelet count, mean platelet volume and thrombocytopoietic indices in healthy women and men. Thromb Res, v. 118, n. 2, p. 199204, 2006

7. CAMERON, H. A. et al. Platelet size in myocardial infarction. Br Med J, v. 287, p. 449-51, 1983.
8. COLLEGE OF AMERICAN PATHOLOGISTS. SURVEYS \& ANATOMIC PATHOLOGY EDUCATION PROGRAMS. Hematology, clinical microscopy and body fluids glossary. 2005. p. 11.

9. DOW, R. B. The clinical and laboratory utility of platelet volume parameters. Aus J Med Sci, v. 15, p. 1-18, 1994.

10. DRACHMAN, J. G. Inherited thrombocytopenia: when a low platelet count does not mean ITP. Blood, v. 103, n. 2, p. 390-8, 2004.

11. ENDLER, G. et al. Mean platelet volume is an independent risk factor for myocardial infarction but not for coronary artery disease. Br J Haematol, v. 117, p. 399-404, 2002.

12. FREEDMAN, J. E. Molecular regulation of platelet-dependent thrombosis. Circulation, v. 112, p. 2725-34, 2005.

13. FUSTER, V. et al. Atherothrombosis and high-risk plaque. JACC, v. 46, n. 6, p. 937-54, 2005.

14. GREISENEGGER, S. et al. Is elevated mean platelet volume associated with a worse outcome in patients with acute ischemic cerebrovascular events? Stroke, v. 35, p. 1688-91, 2004. 
15. GULATI, G. L. et al. Changes in automated complete blood cell count and differential leukocyte count results induced by storage of blood at room temperature. Arch Pathol Lab Med, v. 126, p. 336-42, 2002.

16. HALBMAYER, W. M. et al. Platelet size, fibrinogen and lipoprotein(a) in coronary heart disease. Coronary Artery Disease, v. 6, n. 5, p. 397-402, 1995.

17. HARKER, L. A. et al. Effects of megacaryocyte growth and development factor on platelet production, platelet life span and platelet function in healthy human volunteers. Blood, v. 95, p. 2514-22, 2000.

18. HARTWIG, J.; ITALIANO Jr J. The birth of the platelet. J Thromb Haemost, v. 1, p. 1580-6, 2003.

19. HEKIMSOY, Z. et al. Mean platelet volume in type 2 diabetic patients. J Diabetes Complications, v. 18, p. 173-6, 2004.

20. HUCZEK, Z. et al. Mean platelet volume on admisson predicts impaired reperfusion and long-term mortality in acute myocardial infarction treated with primary percutaneous coronary intervention. JACC, v. 46, n. 2 , p. 284-90, 2005.

21. ITALIANO Jr, J. E.; SHIVDASANI, R. A. Megacaryocytes and beyond: the birth of platelets. J Thromb Haemost, v. 1, p. 1174-82, 2003.

22. KAKKAR, N.; GARG, G. Cytoplasmatic fragments of leukaemic cells masquerading as platelets in an automated haematology analyser. J Clin Pathol, v. 58, p. 224, 2005.

23. KAPSORITAKIS, A. N. et al. Mean platelet volume: a useful marker of inflammatory bowel disease activity. Am J Gastroenterol, v. 96, p. 776-81, 2001.

24. KHANDEKAR, M. M. et al. Platelet volume indices in patients with coronary artery disease and acute myocardial infarction: an Indian scenario. J Clin Pathol, v. 59, p. 146-9, 2006.

25. KILIÇLI-ÇAMUR, N. et al. Could mean platelet volume be a predictive marker for acute myocardial infarction? Med Sci Monit, v. 11, n. 8, p. CR387-392, 2005.

26. KISHK, Y. T; TROWBRIDGE, E. A.; MARTIN, J. F. Platelet volume subpopulations in acute myocardial infarction: an investigation of their homogeneity for smoking, infarct size and site. Clin Sci (Lond), v. 68, n. 4, p. 419-25, 1985.

27. KRISTENSEN, S. D.; MILNER, P. C.; MARTIN, J. F. Bleeding time and platelet volume in acute myocardial infarction: a 2 year follow-up study. Thromb Haemost, v. 59, n. 3, p. 353-6, 1988.

28. MARTIN, J. F.; BATH, P. M. W.; BURR, M. L. Influence of platelet size on outcome after myocardial infarction. Lancet, v. 338, p. 1409-11, 1991.
29. MCBANE, R. D. et al. Platelets characteristics associated with coronary artery disease. J Thromb Haemost, v. 1, p. 1296-303, 2003.

30. MICHELSON, A. D. Platelet function testing in cardiovascular diseases. Circulation, v. 110, p. e489-93, 2004.

31. OSUNA, P. P. et al. Influencia del volumen plaquetario medio sobre el pronóstico a corto plazo del infarto agudo de miocardio. Rev Esp Cardiol, v. 51, p. 816-22, 1998.

32. PIZZULLI, L. et al. Changes in platelet size and count in unstable angina compared to stable angina or non-cardiac chest pain. Eur Heart J, v. 19, p. 804, 1998.

33. SANTOS, E. V.; FILHO, J. M. Plaquetograma em gestantes normais e com pré-eclâmpsia. RBGO, v. 26, n. 3, p. 201-6, 2004.

34. SHAPIRO, A. D. Platelet function disorders. Haemophilia, v. 6, suppl. 1, p. 120-7, 2000.

35. SMITH, N. M.; PATHANSALI, R.; BATH, P. M. W. Platelets and stroke. Vascular Medicine, v. 4, p. 165-72, 1999.

36. VAN DER LOO, B.; MARTIN, J. F. A role for changes in platelet production in the cause of acute coronary syndromes. Arterioscler Thromb Vasc Biol, v. 19, p. 672-9, 1999.

37. VENTURINELLI, M. L. et al. Ativação plaquetária em formas clínicas distintas da doença arterial coronariana (papel da P-selectina e de outros marcadores nas anginas estável e instável). Arq Bras Cardiol, v. 87, p. 446-50, 2006.

38. VOGELAAR, S. A. et al. Blood stability at room temperature for counting red and white blood cells and platelets. Vascul Pharmacol, v. 39, p. 123-5, 2002.

39. YANG, A.; PIZZULLI, L.; LÜDERITZ, B. Mean platelet volume as marker of restenosis after percutaneous transluminal coronary angioplasty in patients with stable and unstable angina pectoris. Thromb Res, v. 117, p. 371-7, 2006.

40. YILMAZ, M. B. et al. Platelet aggregation in left ventricular thrombus formation after acute anterior myocardial infarction: mean platelet volume. Int J Cardiol, v. 90, p. 123-5, 2003.

41. YILMAZ, M. B. et al. Mean platelet volume and exercise stress test. J Thromb Thrombolysis, p. 115-20, 2004.

42. ZAGO, M. A. et al. Hematologia: fundamentos e prática. 1. ed. Ed. São Paulo: Atheneu, 2004. p. 735.

43. ZIMMET, J.; RAVID, K. Polyploidy: occurrence in nature, mechanisms, and significance for the megacaryocyteplatelet system. Experimental Hematology, v. 28, p. 3-16, 2000. 